\title{
Increased serum IL-36 $\beta$ and IL-36 $\gamma$ levels in patients with neuromyelitis optica spectrum disorders: association with disease activity
}

Chun-Sheng Yang ${ }^{1{ }^{*+}} \mathbb{D}$, Qiu xia Zhang ${ }^{1 \dagger}$, Yu Deng ${ }^{1}$, Bing jie Zhou ${ }^{1,2}$, Lin jie Zhang ${ }^{1}$, Li min Li ${ }^{1}$, Yuan Qi ${ }^{1}$, Jing Wang ${ }^{1}$, Li Yang ${ }^{1}$ and Fu-Dong Shi ${ }^{1,3}$

\begin{abstract}
Background: Interleukin 36 (IL-36) cytokines belong to the IL-1 family and play an important role in some autoimmune diseases. However, the relationship between IL-36 and neuromyelitis optica spectrum disorders (NMOSD) remains unclear.

Methods: We determined serum IL-36a, IL-36ß and IL-36y levels and assessed correlations with clinical characteristics in 50 NMOSD patients and 30 healthy controls (HC).

Results: The concentrations of serum IL-36ß and IL-36y were significantly higher in patients with NMOSD than in HCs and decreased during remission. Serum IL-36ß levels were positively correlated with the annual relapse rate (ARR), spinal cord lesion length and Expanded Disability Status Scale (EDSS) scores.
\end{abstract}

Conclusions: Serum IL-36ß and IL-36y levels were related to disease activity in NMOSD patients and may be important biomarkers of NMOSD.

Keywords: Neuromyelitis optica spectrum disorders, Interleukin 36, Biomarkers

\section{Background}

Neuromyelitis optica spectrum disorders (NMOSD) are severe inflammatory disorders of the central nervous system (CNS), which preferentially affect the optic nerve and spinal cord. NMOSD comprise six core clinical characteristics: optic neuritis (ON), acute myelitis (AM), area postrema syndrome, acute brainstem syndrome, acute diencephalic clinical syndrome and symptomatic cerebral syndrome [1]. The discovery of immunoglobulin $\mathrm{G}$ autoantibodies specific for aquaporin-4 (AQP4-IgG) was critical for understanding the pathology of NMOSD $[2,3]$. AQP4-IgG enters the CNS through the damaged blood-brain barrier (BBB), binds to AQP4 on astrocyte

\footnotetext{
* Correspondence: cyang01@tmu.edu.cn

${ }^{+}$Chun-Sheng Yang, Qiu xia Zhang are first two authors contributed equally to this work.

${ }^{1}$ Department of Neurology, Tianjin Neurological Institute, Tianjin Medical University General Hospital, No 154 Anshan Road, Heping District, Tianjin 300052, China

Full list of author information is available at the end of the article
}

endfeet, and activates the complement system, which induces astrocyte damage, granulocyte infiltration, oligodendrocyte death, and neuronal cell death [4].

In addition to AQP4-IgG, cytokines/chemokines, such as IL-6, IL-17, IL-10, IL-12, interferon gamma (IFN- $\gamma$ ), B-cell activating factor (BAFF), CXCL13 and tumour necrosis factor alpha (TNF- $\alpha$ ), may also be involved in the pathogenesis of NMOSD [5-7]. However, the role of newly recognized members of the IL- 1 family, such as IL-36, remains unclear. The IL-36 cytokines include 3

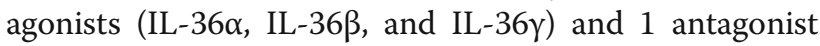
(IL-36Ra); the agonists bind specifically to a common receptor composed of IL-36R and subsequently form a heterodimer with the IL-1R accessory protein (IL1RAcP) to stimulate inflammation [8]. IL-36 cytokines are mainly expressed by keratinocytes, the bronchial epithelium, neuronal cells, glial cells, dendritic cells and macrophages. Recent studies have demonstrated that IL36 is a pro-inflammatory cytokine in several autoimmune 
diseases, including systemic lupus erythematosus (SLE), multiple sclerosis (MS), inflammatory bowel disease, dermatitis, psoriasis, acne and hidradenitis suppurativa [9-15]. However, the possible role of IL-36 in NMOSD is unknown. Therefore, in this study, we determined the serum IL-36 levels of patients with NMOSD and explored the potential relationship between IL-36 and clinical parameters.

\section{Methods}

\section{Patients}

Patients with NMOSD $(n=50)$ who were admitted to Tianjin Medical University General Hospital, Tianjin, China, from December 2015 to December 2018 were recruited. Patients with NMOSD were diagnosed according to the 2015 international consensus diagnostic criteria for NMOSD [1]. Patients who had a history of connective tissue disorders or other immunoinflammatory diseases were excluded. We did not include myelin oligodendrocyte glycoprotein immunoglobulin G (MOG-IgG)-positive patients because the pathophysiology of MOG-IgG-associated NMOSD is probably different from that of AQP4-IgGpositive NMOSD [1]. In the acute phase, all patients with NMOSD were treated with high-dose intravenous methylprednisolone (IVMP) (0.5 g/day for 3-5 consecutive days) and then maintained with low-dose corticosteroids and/or appropriate doses of immunosuppressive agents during remission. In addition, we enrolled 30 healthy age- and sexmatched individuals from the Health Care Center of our hospital.

This study was approved by the ethics committee of Tianjin Medical University General Hospital, and all participants provided written informed consent prior to participation.

\section{Data and sample collection}

The data for demographic and clinical characteristics, age at onset, annual relapse rate (ARR), spinal cord lesion length, and Expanded Disability Status Scale (EDSS) scores at nadir were acquired from medical records. EDSS scores at nadir were evaluated during acute exacerbations before high-dose IVMP by two neurologists.

Fifty serum samples were obtained during acute exacerbations before administration of high-dose IVMP, and 32 serum samples were collected during remission. As a control, $30 \mathrm{HC}$ serum samples were obtained from the Health Care Center of our hospital. All serum samples obtained from participants were stored at $-80^{\circ} \mathrm{C}$ until the time of the assay.

\section{Detection of anti-AQP4 antibodies}

MOG-IgG tests and AQP4-IgG tests were conducted in our clinical neuroimmunological laboratory. As previously described [16], AQP4-IgG was detected by a cell-based assay (CBA).

\section{Measurement of IL-36 levels}

Serum IL-36 $\alpha$, IL-36 $\beta$ and IL-36 $\gamma$ concentrations were

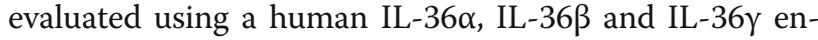
zyme-linked immunosorbent assay (ELISA) kit (R\&D Systems, USA) according to the manufacturer's instructions. Optical densities were measured at $450 \mathrm{~nm}$, and

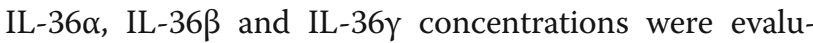
ated with reference to a standard curve. The lowest detectable levels of these cytokines were $12.5 \mathrm{pg} / \mathrm{ml}, 12.5$ $\mathrm{pg} / \mathrm{ml}$ and $18.75 \mathrm{pg} / \mathrm{ml}$, respectively.

\section{Statistical analyses}

Statistical analysis was performed using the Statistical Package for the Social Sciences (SPSS 22.0), and graphs were created using GraphPad Prism 6.01. We applied ttests or the Mann-Whitney $U$ test for quantitative data and the chi-squared test or Fisher's exact test for qualitative data. The relationships between IL-36 and clinical parameters were analysed using Spearman's correlation coefficient. $P$-values $<0.05$ were considered statistically significant for all statistical tests.

\section{Results}

\section{Demographic and clinical characteristics}

The demographic and clinical characteristics of 50 NMOSD patients and $30 \mathrm{HCs}$ are summarized in Table 1.

\section{Serum IL-36 levels}

As shown in Fig. 1, serum IL-36 $\beta$ and IL-36y levels were significantly increased in patients with NMOSD compared to those of $\mathrm{HCs}(P=0.005, P<0.0001$, respectively, Fig. 1a and b), but there was no significant difference in serum IL-36 $\alpha$ levels between the two groups $(P=0.118$, figure not shown). Furthermore, no significant difference in serum IL-36 $\alpha$ and IL-36 $\beta$ levels was found between AQP4-IgG-positive and AQP4-IgG-negative NMOSD patients $(P=0.379$ and 0.141 , respectively, figure not shown). Serum IL-36y levels in AQP4-IgG-negative NMOSD patients were significantly lower than those in AQP4-IgG-positive NMOSD patients $(P=0.040$, Fig. 2$)$ but significantly higher than those in HCs $(P=0.001$, Fig. 2$)$. We also

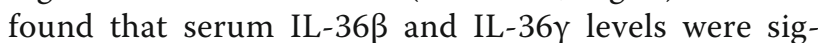
nificantly decreased during the remission period compared to those during the acute phase $(P=$ 0.0001 and 0.013 , respectively, Fig. $3 \mathrm{a}$ and b). Pearson correlation results showed time of immunosuppression wasn't correlated with IL-36 $\beta$ level either in acute or remission phase $(\mathrm{r}=0.242, P=0.183 ; \mathrm{r}=-$ $0.151, P=0.409$, figure not shown). Although there 
Table 1 Demographic and clinical characteristics of NMOSD and HC

\begin{tabular}{|c|c|c|c|}
\hline & $\operatorname{NMOSD}(50)$ & $\mathrm{HC}(30)$ & $P$ \\
\hline Gender, n (\% female) & $48(98 \%)$ & $27(90 \%)$ & 0.358 \\
\hline Age at sampling, years & $46.46 \pm 15.04$ & $51.30 \pm 12.71$ & 0.144 \\
\hline Age at onset, years & $41.9 \pm 15.71$ & - & - \\
\hline Follow-up duration, years & $4.66 \pm 4.33$ & - & - \\
\hline Annualized relapse rate (ARR) & $2.24 \pm 0.97$ & - & - \\
\hline EDSS at nadir & $3.98 \pm 2.12$ & - & - \\
\hline \multicolumn{4}{|l|}{ Presentation at sampling, n (\%) } \\
\hline ON & $7(14 \%)$ & - & - \\
\hline Area postrema syndrome & $2(4 \%)$ & - & - \\
\hline$A M$ & $26(52 \%)$ & - & - \\
\hline Brain stem syndrome & $6(12 \%)$ & - & _ \\
\hline Diencephalic clinical syndrome & $3(6 \%)$ & - & - \\
\hline Cerebral syndrome & $6(12 \%)$ & - & - \\
\hline length of newly identified spinal cord lesion (vertebral segments) & $5(1,15)$ & - & - \\
\hline AQP4-Ab, n (\%) & $28(56 \%)$ & - & - \\
\hline \multicolumn{4}{|l|}{ Imunossupressive agents and dosage, n (\%) } \\
\hline Prednisonlone (12 mg/d) & $5(10 \%)$ & - & - \\
\hline Azathioprine (2 mg/kg.d) & $8(16 \%)$ & - & - \\
\hline Mycophenolate Mofetil(1.5 g/d) & $7(14 \%)$ & - & _ \\
\hline Rituximaba & $30(60 \%)$ & - & \\
\hline
\end{tabular}

Abbreviations: NMOSD neuromyelitis optica spectrum disorders, HC healthy control, ARR annualized relapse rate, EDSS Kurtzke Expanded Disability Status Scale, ON optica neuritis, $A M$ acute myelitis

a: All patients were treated with rituximab (Biogen-Idec, Cambridge,MA, and Genentech, San Francisco, CA) $100 \mathrm{mg}$ (equivalent of 50-59 mg/m²) IV, one infusion per week for 3 consecutive weeks. Continued dosage was dependent on the percentage of circulating CD19 ${ }^{+}$B-cell counts. Whenever it reached $1 \%$ of total lymphocyte population, rituximab $100 \mathrm{mg}$ was reinfused

was correlation between time of immunosuppression and IL36- $\gamma$ level in acute phase $(\mathrm{r}=0.381, P=0.031$, figure not shown), no correlation was found between time of immunosuppression and IL36- $\gamma$ level in remission phase $(\mathrm{r}=0.117, \quad P=0.525$, figure not shown). Serum IL-36 $\alpha$ levels were lower during the remission period than during the acute phase in NMOSD patients, but this difference did not reach the level of statistical significance $(P=0.342$, figure not shown).
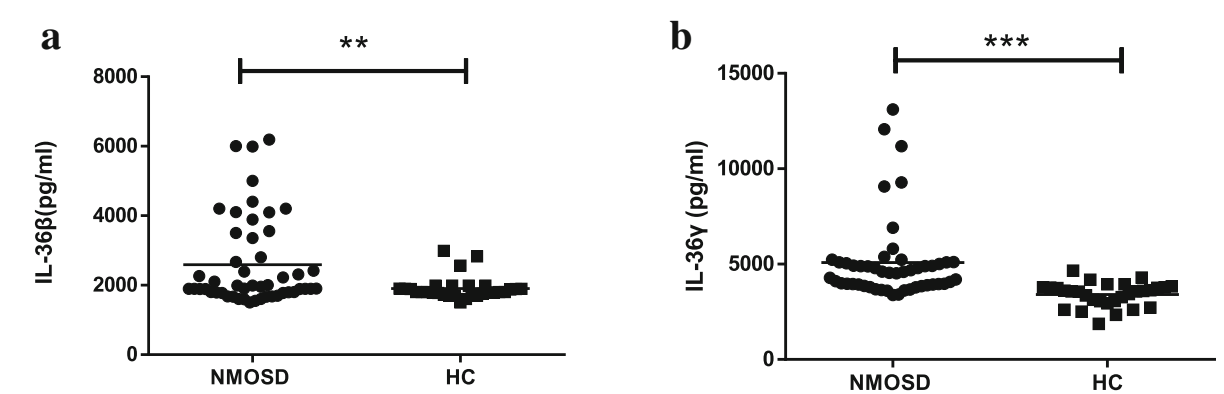

Fig. 1 Serum IL-36ß and IL-36y levels in NMOSD patients and HC. NMOSD = neuromyelitis optica spectrum disorders; HC = healthy controls. a Comparison of serum IL-36ß levels in NMOSD patients and HC. b Comparison of serum IL-36y levels in NMOSD and HC. ${ }^{* *} P<0.01$, *** $P<0.001$ 


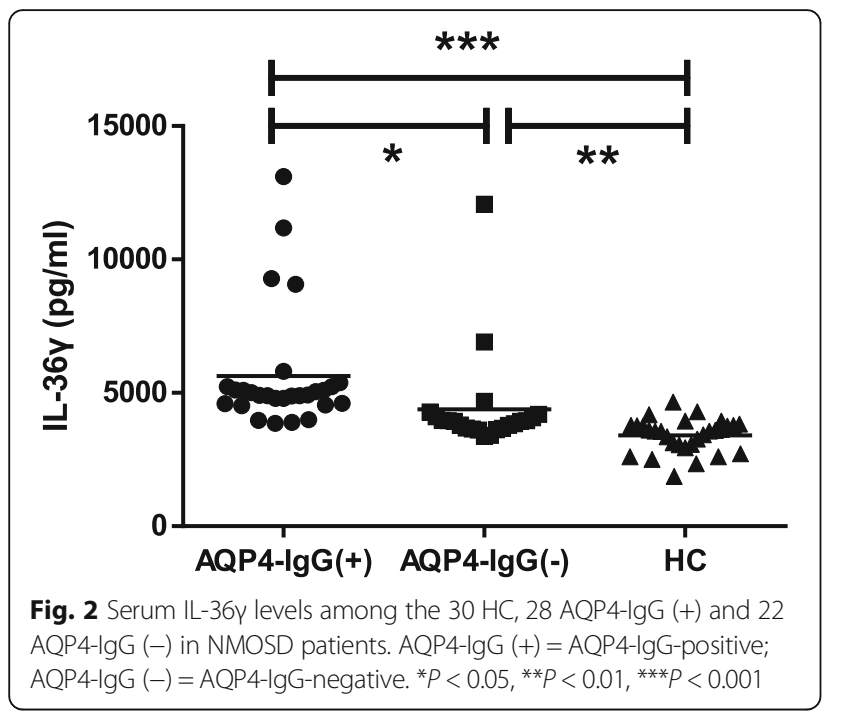

\section{Correlations between serum IL-36 levels and clinical parameters}

We performed a correlation analysis between serum IL36 levels and clinical parameters to explore potential associations. The relationship between ARR and serum IL-36 levels for 44 NMOSD patients is shown in Fig. 4; the other six NMOSD patients were excluded because the follow-up duration was less than 3 months. A significant positive correlation was observed between serum IL-36 $\beta$ levels and ARR $(r=0.698, P<0.0001$, Fig. 4a), while neither serum IL-36 $\alpha$ levels nor serum IL-36y levels were correlated with ARR in patients with NMOSD $(r=0.087, P=0.574 ; r=0.136, P=0.378$, figure not shown). Considering that the EDSS scores of patients with different clinical syndromes of NMOSD may vary during acute exacerbations, we enrolled 26 NMOSD patients who presented with acute attacks of AM for further study. We found that serum IL-36 $\beta$ levels were positively correlated with the length of newly identified spinal cord lesions and the EDSS scores at nadir in patients with NMOSD patients $(r=0.613, P<$ 0.001 , Fig. 4b; $r=0.426, P=0.030$, Fig. 4c, respectively). Furthermore, neither serum IL-36 $\alpha$ levels nor serum IL-
$36 \gamma$ levels correlated with the length of newly identified spinal cord lesions $(\mathrm{r}=0.116, P=0.571 ; \mathrm{r}=0.129, P=$ 0.529 , respectively, figure not shown). We found that there were no significant correlations between serum IL$36 \alpha$ levels and EDSS scores $(r=-0.011, P=0.957$, figure not shown) or serum IL-36y levels and EDSS scores $(r=$ $0.005, P=0.980$, figure not shown).

\section{Discussion}

Previous studies have reported increased serum IL-36 levels in patients with autoimmune disorders, and serum IL-36 may be involved in the pathological process associated with autoimmune diseases. Emerging data have suggested that IL-36 is a pro-inflammatory signal that triggers further inflammatory mediators and a potential therapeutic target for the treatment of immune diseases in humans $[14,15]$. In the present study, we first dem-

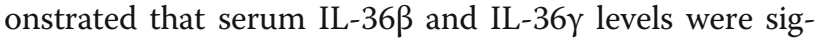
nificantly increased in patients with NMOSD compared to those in $\mathrm{HC}$, especially in the acute stage, suggesting that IL-36 might play an important role in the inflammatory pathogenesis of NMOSD. Recent research indicates that serum IL-36 $\alpha$ and IL-36y levels are markedly increased in SLE patients and are strongly correlated with SLE Disease Activity Index (SLEDAI) scores and complement C3 levels [16]. Furthermore, IL-36y levels are elevated in patients with atopic dermatitis and have proinflammatory effects on human endothelial cells $[10,17]$. In addition, a recent report showed that IL-36 $\alpha$ and IL$36 \gamma$ may play a pro-inflammatory role in the pathophysiology of inflammatory bowel disease [9]. The present

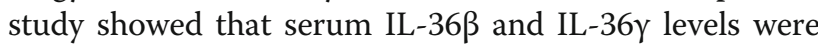
significantly decreased during the remission period compared to those in the acute phase. Serum IL-36 $\beta$ levels were positively correlated with ARR, the length of newly identified spinal cord lesions and the EDSS scores at nadir. IL-36 $\beta$ is associated with the severity of disease in patients with NMOSD. These findings indicated that IL36 was associated with disease activity of NMOSD patients. Further studies are required to investigate the possible role of IL -36 in NMOSD. Despite an
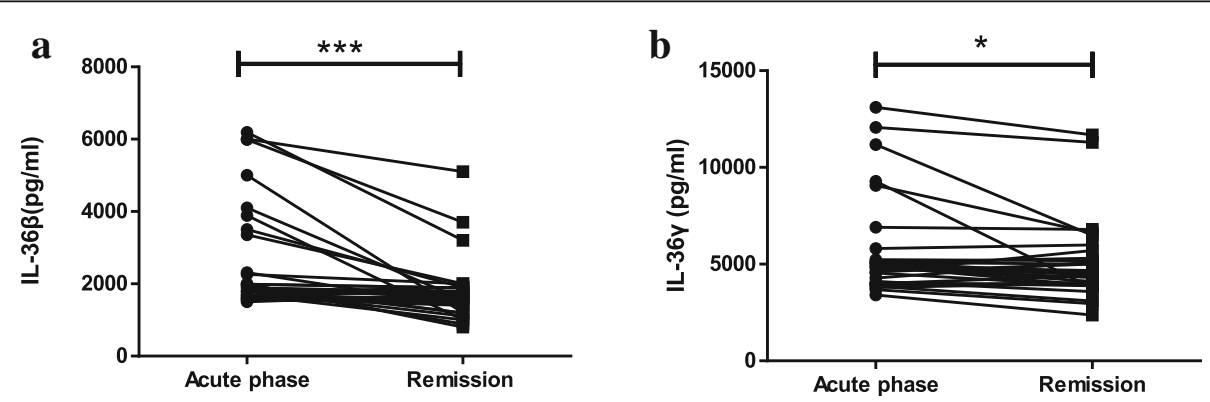

Fig. 3 Serum IL-36ß and IL-36y levels in the acute and remission phases in 32 NMOSD patients. a Comparison of serum IL-36ß levels between acute and remission in NMOSD patients. b Comparison of serum IL-36y levels between acute and remission in NMOSD patients. ${ }^{*} P<0.05$, ${ }^{* * *} P<0.001$ 

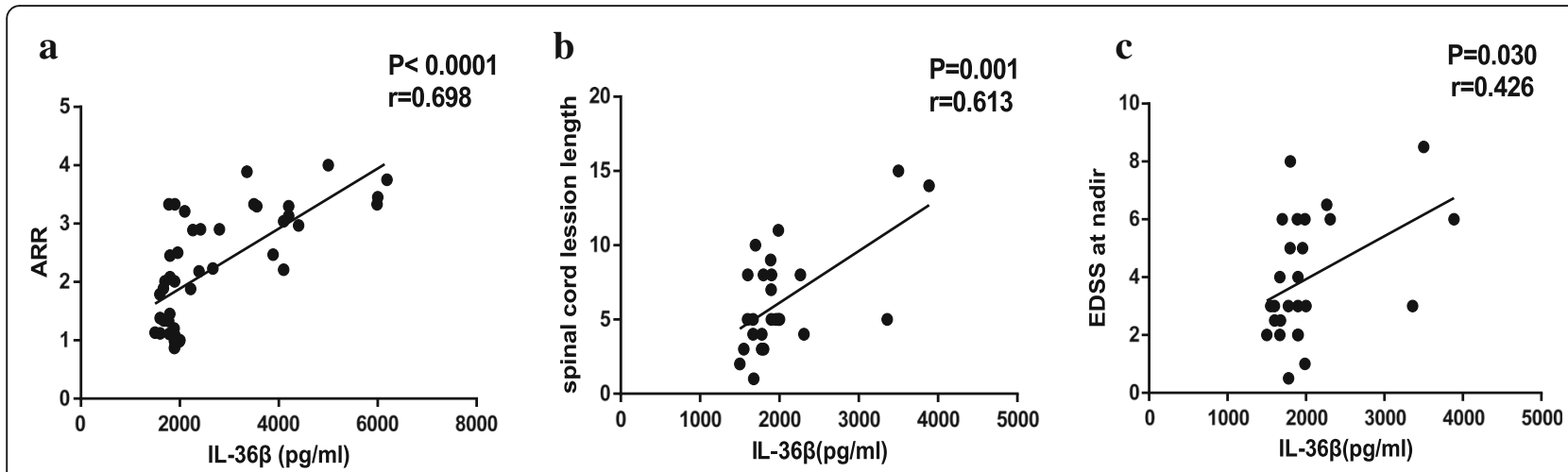

Fig. 4 Correlation between serum IL-36ß levels and clinical characteristics in NMOSD patients. a Correlation between serum IL-36ß levels and ARR in 44 NMOSD patients. ARR = annual relapse rate. $\mathbf{b}$ Correlation between serum IL-36ß levels and spinal cord lession length in 26 NMOSD patients. c Correlation between serum IL-36ß levels and EDSS at nadir in 26 NMOSD patients. EDSS = Expanded Disability Status Scale

increasing number of studies on the pathophysiology and signalling pathways associated with IL-36 during the immune response, few breakthroughs have been reported. Previous studies suggest that IL-36 $\alpha$, IL-36 $\beta$ and IL-36y signal through IL-36R and IL-1RAcP to activate a pathway that leads to NF- $\mathrm{kB}$ and MAPKs $[5,18,19]$. In addition, IL-36 potently stimulates human M2 macrophages, Langerhans cells and keratinocytes to produce pro-inflammatory cytokines [20]. However, the pathophysiological mechanism and signalling pathway of IL36 in NMOSD remain unclear.

A previous study showed that IL-36y is upregulated in models of experimental autoimmune encephalitis (EAE) and that microglia are a potential target of IL-36y [21]. Furthermore, IL-36y derived from neutrophils could stimulate microglia to produce neutrophil-stimulating cytokines [21]. This finding was consistent with those studies, which showed that IL-36y induced both the production of such cytokines in other myeloid cells residing outside the CNS and the recruitment of neutrophils [19, 22-24]. Neutrophils play a prominent role in the pathogenesis of NMOSD because abnormal neutrophil aggregation was found in NMOSD lesions [3, 25], and neutrophil protease inhibition reduces AQP4-IgG damage in the mouse brain [26]. Yang reported increased plasma levels of epithelial neutrophil-activating (ENA) peptide $78 /$ CXCL5 during the remission of NMOSD [27]. That study showed that ENA 78 plasma levels correlated positively with EDSS scores in NMOSD patients. The overproduction of pro-inflammatory cytokines, such as IL-1 $\beta$ activates, ENA 78, which in turn leads to neutrophil infiltration into lesions. All these findings suggest that IL-36y could contribute to neuroinflammation, perhaps by promoting neutrophil recruitment.

Rafael reported that the IL-36 receptor was expressed by human blood and intestinal $\mathrm{T}$ lymphocytes and was activated via IL-36 $\beta$ in a dose-dependent manner, inducing proliferation of $\mathrm{CD} 4+\mathrm{T}$ lymphocytes [28]. The direct induction of IL-17A by IL-36 agonists was observed in cultured murine CD4+ T cells [22]. A study also showed that Th17 cytokines, such as IL-17A, directly induced IL-36 cytokines and in turn enhanced their own expression and the production of proinflammatory cytokines, such as IL-6 and IL-8, in cultured human keratinocytes, forming a positive feedback loop between IL-36 and Th17 cytokines [29]. Many studies have also shown increased CSF IL-6 and IL-17 levels in patients with NMO [30-32]. Th17- and Th2-related cytokines are upregulated in the CSF of NMO patients [31]. All of these results indicated that IL-36 could play an important role in the pathogenesis of NMOSD.

This study had certain limitations. First, as it is retrospective, bias is inevitable. Second, the patients were insufficient in number without a control group with another CNS autoimmune disease, for example MS. In the future, we will recruit more NMOSD patients in a perspective study with a control group with another CNS autoimmune disease, which may lead to a deeper understanding of the role of IL-36 in NMOSD.

\section{Conclusions}

The present study first showed that serum IL-36 $\beta$ and IL$36 \gamma$ levels were increased in NMOSD patients, especially during the acute stage. Serum IL-36 $\beta$ levels were positively correlated with ARR, spinal cord lesion length and EDSS scores. Due to the small sample and the observational nature of this study, additional research on larger samples is needed to investigate pathological mechanisms and signalling pathways related to IL-36 in NMOSD.

\section{Abbreviations}

AM: Acute myelitis; AQP4-IgG: Immunoglobulin G autoantibodies specific for aquaporin-4; ARR: Annual relapse rate; BAFF: B-cell activating factor; BBB: Blood-brain barrier; CBA: Cell-based assay; CNS: Central nervous system; EAE: Experimental autoimmune encephalitis; EDSS: Expanded Disability Status Scale; ELISA: Enzyme-linked immunosorbent assay; ENA: Epithelial neutrophilactivating; HC: Healthy controls; IFN-ү: Interferon gamma; IL: Interleukin; IL- 
1RacP: IL-1R accessory protein; IVMP: High-dose intravenous methylprednisolone; MOG-IgG: Myelin oligodendrocyte glycoprotein immunoglobulin G; MS: Multiple sclerosis; NMOSD: Neuromyelitis optica spectrum disorders; ON: Optic neuritis; SLE: Systemic lupus erythematosus; SLEDAI: SLE Disease Activity Index; SPSS: Statistical Package for the Social Sciences; TNF-a: Tumour necrosis factor alpha

\section{Acknowledgements}

We thank Drs A. Vincent and D. Beeson for providing plasmids for AQP4, and we thank our patients for participating in this study.

\section{Authors' contributions}

CSY, LY and FDS participated in study design. QXZ, YD, BJZ, LJZ, LML, YQ and JW participated in data collection. CSY and QXZ participated in statistical analysis. All authors read and approved the final manuscript.

\section{Funding}

The study was supported by the National Natural Science Foundation of China (grant number: 81571172, 81771363)

\section{Availability of data and materials}

The datasets used and/or analyzed during the current study are available from the corresponding author on reasonable request.

\section{Ethics approval and consent to participate}

The study was approved by the Ethics Committee of Tianjin Medical University General Hospital, and written informed consent was obtained from each participant. This study was performed in accordance with the Declaration of Helsinki and all authors agreed the publish statements of BMC Neurology.

\section{Consent for publication}

Written informed consent for publication of their clinical details and clinical images was obtained from all the patients. A copy of the consent form is available for review by the Editor of this journal.

\section{Competing interests}

All authors declare that they have no competing interest.

\section{Author details}

${ }^{1}$ Department of Neurology, Tianjin Neurological Institute, Tianjin Medical University General Hospital, No 154 Anshan Road, Heping District, Tianjin 300052, China. ${ }^{2}$ Department of Neurology, Tianjin TEDA Hospital, No 65 The Third Road, Tianjin Economic Technological Development Area, Tianjin 300457, China. ${ }^{3}$ Department of Neurology, Barrow Neurological Institute, St. Joseph's Hospital and Medical Center, Phoenix, AZ 85013, USA.

Received: 26 January 2019 Accepted: 25 July 2019

Published online: 05 August 2019

\section{References}

1. Wingerchuk DM, Banwell B, Bennett JL, Cabre P, Carroll W, Chitnis T, et al. International consensus diagnostic criteria for neuromyelitis optica spectrum disorders. Neurology. 2015;85:177-89.

2. Lennon VA, Kryzer TJ, Pittock SJ, Verkman AS, Hinson SR. IgG marker of optic-spinal multiple sclerosis binds to the aquaporin-4 water channel. J Exp Med. 2005;202:473-7.

3. Lennon VA, Wingerchuk DM, Kryzer TJ, Pittock SJ, Lucchinetti CF, Fujihara K, et al. A serum autoantibody marker of neuromyelitis optica: distinction from multiple sclerosis. Lancet. 2004;364:2106-12.

4. Papadopoulos MC, Verkman AS. Aquaporin 4 and neuromyelitis optica. Lancet Neurol. 2012:11:535-44.

5. Matsushita T, Tateishi T, Isobe N, Yonekawa T, Yamasaki R, Matsuse D, et al. Characteristic cerebrospinal fluid cytokine/chemokine profiles in neuromyelitis optica, relapsing remitting or primary progressive multiple sclerosis. PLoS One. 2013;8:e61835.

6. Uzawa A, Mori M, Kuwabara S. Cytokines and chemokines in neuromyelitis optica: pathogenetic and therapeutic implications. Brain Pathol. 2014;24:67-73.
7. Kothur K, Wienholt L, Brilot F, Dale RC. CSF cytokines/chemokines as biomarkers in neuroinflammatory CNS disorders: a systematic review. Cytokine. 2016;77:227-37.

8. Gunther S, Sundberg EJ. Molecular determinants of agonist and antagonist signaling through the IL-36 receptor. J Immunol. 2014;193:921-30.

9. Chu M, Wong CK, Cai Z, Dong J, Jiao D, Kam NW, et al. Elevated expression and pro-inflammatory activity of IL-36 in patients with systemic lupus erythematosus. Molecules. 2015;20:19588-604.

10. Alsahebfosoul F, Jahanbani-Ardakani H, Ghavimi R, Sedaghat N, Etemadifar M. Serum level of interleukin 36 in patients with multiple sclerosis. J Immunoassay Immunochem. 2018;39:558-64.

11. Russell SE, Horan RM, Stefanska AM, Carey A, Leon G, Aguilera M, et al. IL36alpha expression is elevated in ulcerative colitis and promotes colonic inflammation. Mucosal Immunol. 2016;9:1193-204.

12. Nishida A, Hidaka K, Kanda $T$, Imaeda H, Shioya M, Inatomi $O$, et al. Increased expression of interleukin-36, a member of the interleukin-1 cytokine family, in inflammatory bowel disease. Inflamm Bowel Dis. 2016;22:303-14

13. Otobe S, Sugaya M, Nakajima R, Oka T, Takahashi N, Kabasawa M, et al. Increased interleukin-36gamma expression in skin and sera of patients with atopic dermatitis and mycosis fungoides/sezary syndrome. J Dermatol. 2018; 45:468-71.

14. Mahil SK, Catapano M, Di Meglio P, Dand N, Ahlfors H, Carr IM, et al. An analysis of IL-36 signature genes and individuals with IL1RL2 knockout mutations validates IL-36 as a psoriasis therapeutic target. Sci Transl Med. 2017:9:eaan2514.

15. Di Caprio R, Balato A, Caiazzo G, Lembo S, Raimondo A, Fabbrocini G, et al. IL-36 cytokines are increased in acne and hidradenitis suppurativa. Arch Dermatol Res. 2017;309:673-8.

16. Yang CS, Zhang DQ, Wang JH, Jin WN, Li MS, Liu J, et al. Clinical features and sera anti-aquaporin 4 antibody positivity in patients with demyelinating disorders of the central nervous system from Tianjin, China. CNS Neurosci Ther. 2014;20:32-9.

17. Bridgewood C, Stacey M, Alase A, Lagos D, Graham A, Wittmann M. IL36gamma has proinflammatory effects on human endothelial cells. Exp Dermatol. 2017:26:402-8.

18. Dunn E, Sims JE, Nicklin MJ, O'Neill LA. Annotating genes with potential roles in the immune system: six new members of the IL-1 family. Trends Immunol. 2001:22:533-6.

19. Towne JE, Garka KE, Renshaw BR, Virca GD, Sims JE. Interleukin (IL)-1F6, IL1F8, and IL-1F9 signal through IL-1Rrp2 and IL-1RACP to activate the pathway leading to NF-kappaB and MAPKs. J Biol Chem. 2004;279:13677-88.

20. Dietrich D, Martin P, Flacher V, Sun Y, Jarrossay D, Brembilla N, et al. Interleukin-36 potently stimulates human M2 macrophages, Langerhans cells and keratinocytes to produce pro-inflammatory cytokines. Cytokine. 2016;84:88-98.

21. Bozoyan L, Dumas A, Patenaude A, Vallieres L. Interleukin-36gamma is expressed by neutrophils and can activate microglia, but has no role in experimental autoimmune encephalomyelitis. J Neuroinflammation. 2015;12:173

22. Vigne S, Palmer G, Lamacchia C, Martin P, Talabot-Ayer D, Rodriguez E, et al. IL-36R ligands are potent regulators of dendritic and T cells. Blood. 2011 118:5813-23.

23. Foster AM, Baliwag J, Chen CS, Guzman AM, Stoll SW, Gudjonsson JE, et al. IL-36 promotes myeloid cell infiltration, activation, and inflammatory activity in skin. J Immunol. 2014;192:6053-61.

24. Ramadas RA, Ewart SL, Medoff BD, LeVine AM. Interleukin-1 family member 9 stimulates chemokine production and neutrophil influx in mouse lungs. Am J Respir Cell Mol Biol. 2011:44:134-45.

25. Papadopoulos MC, Bennett JL, Verkman AS. Treatment of neuromyelitis optica: state-of-the-art and emerging therapies. Nat Rev Neurol. 2014;10:493-506.

26. Saadoun $S$, Waters $P$, MacDonald C, Bell BA, Vincent A, Verkman AS, et al. Neutrophil protease inhibition reduces neuromyelitis optica-immunoglobulin G-induced damage in mouse brain. Ann Neurol. 2012;71:323-33.

27. Yang T, Wang S, Zheng Q, Wang L, Li Q, Wei M, et al. Increased plasma levels of epithelial neutrophil-activating peptide 78/CXCL5 during the remission of Neuromyelitis optica. BMC Neurol. 2016;16:96.

28. Penha R, Higgins J, Mutamba S, Barrow P, Mahida Y, Foster N. IL-36 receptor is expressed by human blood and intestinal T lymphocytes and is dosedependently activated via IL-36beta and induces CD4+ lymphocyte proliferation. Cytokine. 2016;85:18-25. 
29. Carrier Y, Ma HL, Ramon HE, Napierata L, Small C, O'Toole M, et al. Interregulation of Th17 cytokines and the IL-36 cytokines in vitro and in vivo: implications in psoriasis pathogenesis. J Invest Dermatol. 2011;131:2428-37.

30. Wang H, Wang K, Wang C, Xu F, Zhong X, Qiu W, et al. Cerebrospinal fluid high-mobility group box protein 1 in neuromyelitis optica and multiple sclerosis. Neuroimmunomodulation. 2013;20:113-8.

31. Uzawa A, Mori M, Arai K, Sato Y, Hayakawa S, Masuda S, et al. Cytokine and chemokine profiles in neuromyelitis optica: significance of interleukin-6. Mult Scler. 2010;16:1443-52.

32. Barros PO, Cassano T, Hygino J, Ferreira TB, Centuriao N, Kasahara TM, et al. Prediction of disease severity in neuromyelitis optica by the levels of interleukin (IL)-6 produced during remission phase. Clin Exp Immunol. 2016; 183:480-9.

\section{Publisher's Note}

Springer Nature remains neutral with regard to jurisdictional claims in published maps and institutional affiliations.

Ready to submit your research? Choose BMC and benefit from:

- fast, convenient online submission

- thorough peer review by experienced researchers in your field

- rapid publication on acceptance

- support for research data, including large and complex data types

- gold Open Access which fosters wider collaboration and increased citations

- maximum visibility for your research: over $100 \mathrm{M}$ website views per year

At $B M C$, research is always in progress.

Learn more biomedcentral.com/submissions 